
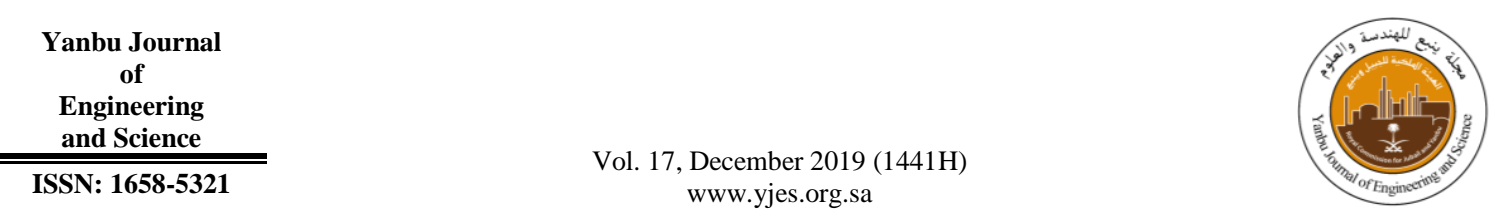

\title{
INVESTIGATION OF FLUID STRUCTURE INTERACTION (FSI) EFFECT ON A FRP (FIBER REINFORCED POLYMER) POLE USING ANSYS
}

\author{
Anand Valiavalappil \\ Advanced Computational Laboratory of Yanbu Research Center \\ Department of Mechanical Engineering Technology, Yanbu Industrial College, Kingdom of Saudi Arabia \\ E-mail: valiavalappila@rcyci.edu.sa
}

\begin{abstract}
This paper presents a simulation study on a fiber-reinforced polymer (FRP) light pole that is being used in Yanbu Industrial City, Saudi Arabia. The light poles in this area are subjected to cyclic wind load blowing from the Red Sea. It is of great interest to re-investigate the strength and integrity of the structure for future improvement. A three-dimensional numerical simulation has been performed to analyze the effect of wind velocity over the FRP pole. The pole is conical in shape, having a circular hollow cross-section in both the ends and tapered uniformly. The pole considered for simulation is having a height of 6 meters and have conicity of $18 \mathrm{~mm} / \mathrm{m}$. The FRP pole is a combination of unsaturated polyester, vinyl ester, epoxy, phenolic, polyurethane, and glass fibers, which are reinforced by the centrifugal process. The computational fluid dynamics (CFD) simulation results are validated with the mathematical calculation. The adopted method is fluid-structure interaction (FSI) with SST $k-\omega$ (shear stress transport) turbulence model, which is used to simulate the wind velocity over the FRP pole using ANSYS Fluent. The velocity of air considered for simulation ranges from 10 to $50 \mathrm{~m} / \mathrm{sec}$. The study provided a good correlation between mathematical calculation and the CFD simulation for each air velocity. The study revealed maximum deflection and equivalent stress occurred at the top and bottom end, which are $217 \mathrm{~mm}$ and $85 \mathrm{MPa}$ respectively. The exact region of crack is also predicted for different wind velocity. This method can also be adopted for any FRP poles available.
\end{abstract}

\section{Keywords: Street Light Pole; CFD (Computational fluid Dynamics); Structural analysis using ANSYS (CFD \& FEA)}

\section{INTRODUCTION}

The light pole started its journey as a wood material, later it was replaced by the steel. The light pole are generally made of wood, steel, and FRP material. The latest type of material used for manufacturing of light pole is FRP (fiber-reinforced polymer) material. The reinforcement within the FRP pole makes it better in strength and stiffness, also the resins creates a stronger bond for the fibers within the pole. The recent development in material properties of FRP, have made it better than other materials used to manufacture light pole. The main properties FRP includes corrosion resistant, light weight and high tensile strength [1]. This properties of FRP makes it a perfect material for street light. The corrosion resistant makes the pole to have a longer life with a less maintenances requirement. The FRP pole provides a better absorption energy during the impact [2]. This 
advantage makes it safer for cars during any collision with the street light pole made of FRP. The FRP pole are manufactured using various techniques, such as filament winding, pultrusion and centrifugal casting. The pole used for simulation process is manufactured using the rotational mould method. This method uses a casting technique in which the material quantity for a closed mould is less than the total volume. The remaining volume is compensated by rotating the chamber to have a coated surface until the resin get settled. This provides an advantage of hollow portion within the pole and thereby material reduction. There are different kinds of design used in pole for the street light application. According to the design, force exerted over the pole surface will vary. The variation depends upon the air velocity and is directly proportional to pole deflection. The deflection depends upon the design of pole, extender and lamp. This highlight's that the total wind load is sum total of wind velocity against pole, extender and lamp of the street light. The ANSYS Predicts a very accurate results using the turbulence model for external flow simulation. The turbulences model used for the simulation is SST $k-\omega$ (shear stress transport) turbulence. This model predicts the accurate force exerted over the pole for different wind velocity. This force will be further applied over the pole using FSI technique, thus the deflection and crack occurring region can be accurately predicted.

\section{LIGHT POLE DESCRIPTION AND PARAMETERS}

The light pole used in street light have a height of $6000 \mathrm{~mm}$ and the distances between extenders are $2000 \mathrm{~mm}$. The arm extender with luminaire have a gross mass of $17 \mathrm{~kg}$, arm extender is made up of steel pipe also the electrical door have a length of $290 \mathrm{~mm}$. The pole have a foundation made of reinforced concrete with a steel plate for anchoring. The bolt will be anchoring the steel plate with the ground, and the FRP pole is attached with the steel plate, in Figure 1.

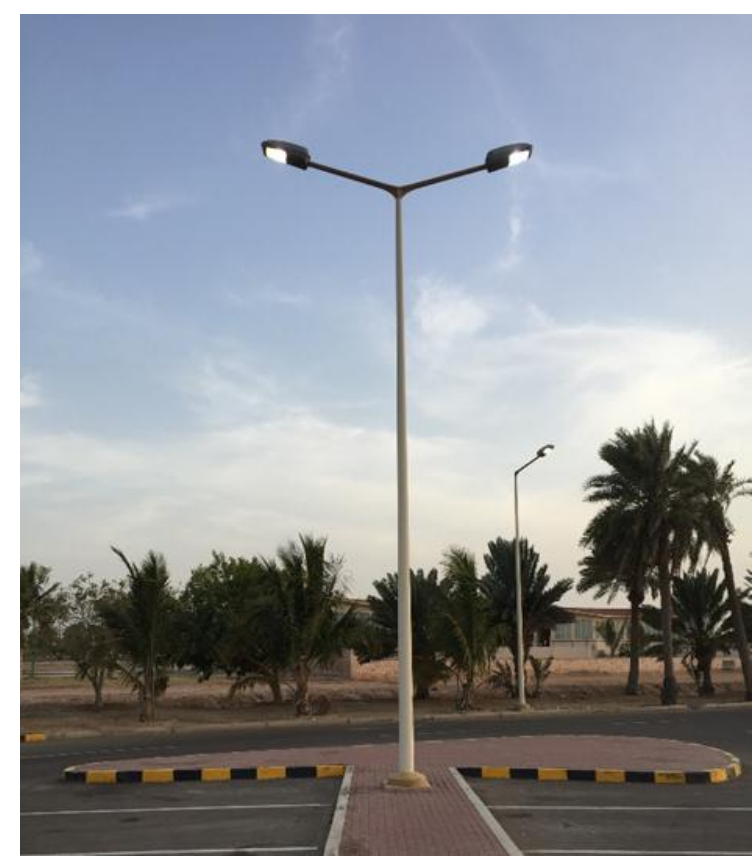

Fig. 1. Street light pole in Yanbu KSA (Shairco Company)

\subsection{MATERIAL PROPERTIES}

Mechanical properties of FRP for the pole used in street light used in Yanbu, K.S.A. Tensile strength $=250 \mathrm{~N} / \mathrm{mm}^{2}$, Flexural strength $=170 \mathrm{~N} / \mathrm{mm}^{2}$, Flex. E - Modulus $=7000 \mathrm{~N} / \mathrm{mm}^{2}$, Impact strength $5 \mathrm{~N} / \mathrm{mm}^{2}$, Density of FRP $=(0.055$ ib/inch3 $)$ or 1522 $\mathrm{kg} / \mathrm{m} 3$. The structural property of steel is already available in ANSYS database.

\section{METHODOLOGY}

The modelling of the CAD is performed and it's exported to ANSYS Fluent. The fluent is used to find out the pressure exerted over the surface. The inlet velocity applied is 200 $\mathrm{km} / \mathrm{hr}$, and external flow simulation methodology is adopted.

\subsection{THE WORKING OF A CFD CODE}

All the CFD codes works on a numerical algorithms and which have the capability of tackling the fluid flow problems. All the commercially available software have their user interface with regards to input / output results. [5] The CFD is an instrumental part 
of three major equations, which are NavierStokes, continuity and energy equations [6]. The CFD creates the simulation based study upon various flow pattern, and also it have a high impact on the complex aerodynamics designs [7]. The simulation is based on external flow, which is also enhanced with Fluid structure interaction. The fluid structure interaction provides a very strong output in terms of results [8].

Following are the division for CFD working procedure:

1. Preprocessing: This introduce the definition of geometry, Grid/mesh generation, type of fluid required (Air, Water) etc. It also gives an option for creating the required boundary conditions for solving the problem.

2. Solver: The solver have three methods of approach Finite differences, finite element and finite volume method. The main differences between them are flow approximation and discrete method used. In this simulation we are using ANSYS fluent and which works on control volume method.

3. Post processing: It is the final part of $\mathrm{CFD}$, in which we get the results in terms on contour (Color Plot), particle tracking etc.

\subsection{NUMERICAL METHOD}

\subsubsection{Governing equations}

The simulation is carried out using the Navier-Stoke equation for an external flow simulation. The mass conservation equation is also the continuity equation. It is defined as the rate of change of mass within a control volume $(\mathrm{CV})$ is equivalent to the net rate of mass flowing into the Control volume. [9].

$$
\frac{\partial p}{\partial t}+\frac{\partial(\rho u)}{\partial x}+\frac{\partial(\rho v)}{\partial y}+\frac{\partial(\rho w)}{\partial z}=0
$$

Navier stoke equation for $\mathrm{X}, \mathrm{Y}$ and $\mathrm{Z}$ direction are as follows:

$$
\begin{aligned}
\frac{\partial(p u)}{\partial t}+\nabla(\rho u V)= & -\frac{\partial \rho}{\partial x}+\frac{\partial \tau x x}{\partial x}+\frac{\partial \tau y x}{\partial y}+\frac{\partial \tau z x}{\partial z}+\rho f x \\
& (\text { X direction }) \\
\frac{\partial(p v)}{\partial t}+\nabla(\rho v V)= & -\frac{\partial \rho}{\partial x}+\frac{\partial \tau y x}{\partial x}+\frac{\partial \tau y y}{\partial y}+\frac{\partial \tau z y}{\partial z}+\rho f y \\
& (\mathrm{Y} \text { direction) } \\
\frac{\partial(p u)}{\partial t}+\nabla(\rho w V)= & -\frac{\partial \rho}{\partial x}+\frac{\partial \tau x x}{\partial x}+\frac{\partial \tau y x}{\partial y}+\frac{\partial \tau z x}{\partial z}+\rho f x
\end{aligned}
$$$$
\text { (Z direction) }
$$

The following are the boundary conditions applied.

1. Inlet: velocity $200 \mathrm{~km} / \mathrm{hr}$, Outlet: Pressure outlet

2. Density based method $\left(\rho=1.225 \mathrm{~kg} / \mathrm{m}^{3}\right)$

3. Viscous model is selected to be Standard K - Omega (2 equation), Gravity is specified along the pole.

\subsubsection{Design Calculation}

The pole is having a circular cross section, and the wind velocity is considered to be $195 \mathrm{~km} / \mathrm{hr}$. The risk co-efficient $k 1=1.05$ (Structure life is calculated for hundred years). The terrain factor $k 2$ according to the pole height is 0.98 . The topography factor $k 3$ is taken to be 1 [12]. The total wind load is considered to be:

$=\mathrm{k} 1 \times \mathrm{k} 2 \times \mathrm{k} 3 \mathrm{x}$ wind velocity [13]

$=200 \mathrm{~km} / \mathrm{hr} \approx(55 \mathrm{~m} / \mathrm{sec})$

The pole is having a mass of $27 \mathrm{~kg}$ or a weight of $265 \mathrm{~N}$. The pole have a top diameter of $76 \mathrm{~mm}$, and the base diameter of $184 \mathrm{~mm}$, with a height of $6000 \mathrm{~mm}(6 \mathrm{~m})$. The wind load $=0.4 \mathrm{X}$ Total wind load $=1210 \mathrm{~N}$ [13]. The total height of Pole is $6 \mathrm{~m}$, or $6000 \mathrm{~mm}$, the load is considered to be UDL (Uniformly distributed Load), so the load is $(1210 / 6000)=0.201 \mathrm{~N} / \mathrm{mm}$ [14]

Moment of inertia $(\mathrm{t})=\frac{\pi}{64}\left(184^{4}-164^{4}\right)=20755811 \mathrm{~mm}^{4}$

Modulus of elasticity $(E)=7000 \mathrm{~N} / \mathrm{mm}^{2}$ 
MaximumDeflection $=\frac{\left(W^{4}\right)}{8 E I}=\frac{\left(0.201 \times 6000^{4}\right)}{8(7000) \times(28656237)}=224 \mathrm{~mm}$

(Where $\mathrm{W}$ is the load for UDL, 1 is the length or height for the pole, Modulus of elasticity for the fiber reinforced pole, Moment of inertia for the circular cross section (I))

\section{COMPUTATIONAL DOMAIN AND RESULTS ANALYSIS USING (ANSYS FLUENT/STRUCTURAL ANALYSIS)}

The model for the Street light is shown in the Figure 2 below. It has a four main features, which are pole, extender, anchoring part and illuminator.

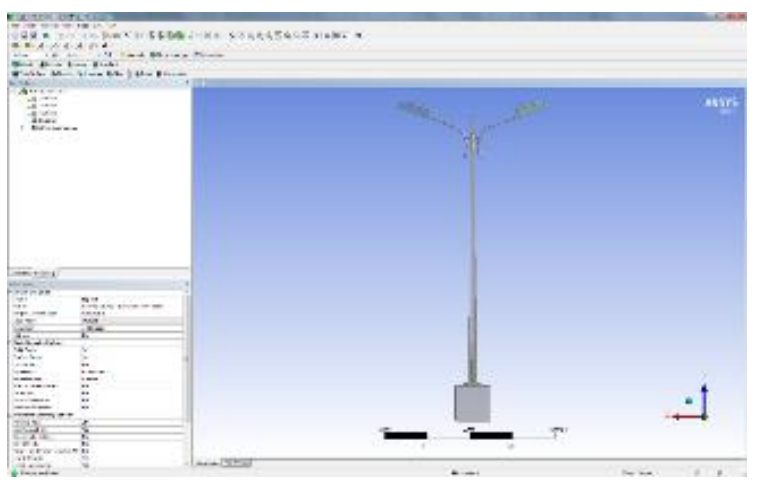

Fig. 2. The cad diagram for Street light

The ANSYS (Fluent) is performed, producing a result of Force created over the pole surface. The force is 1173 Newton, which compared to the hand calculation results a very closer value $(1210 \mathrm{~N})$. In this simulation work, the pole is only considered to be applying the required air velocity, Figure 3.

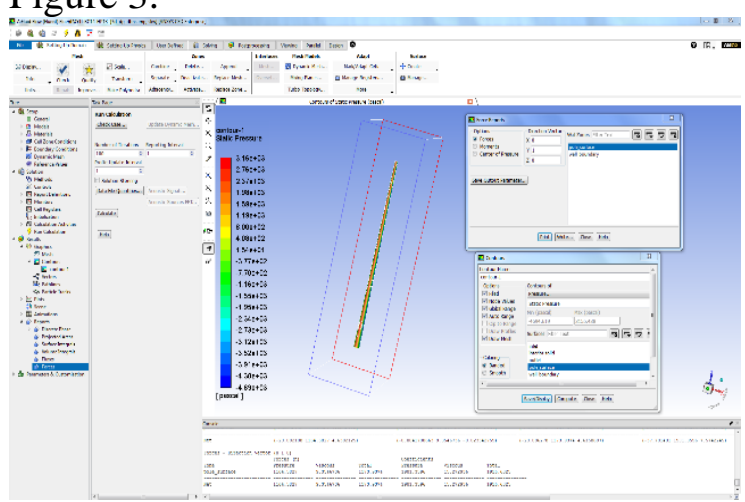

Fig. 3. The Ansys fluent simulation result
The Value obtained from the ANSYS simulation is carried out further to the Static Structural analysis. The coupling creates a FSI (Fluid Structure Interaction) between the fluid and solid region in contact, Figure 4.

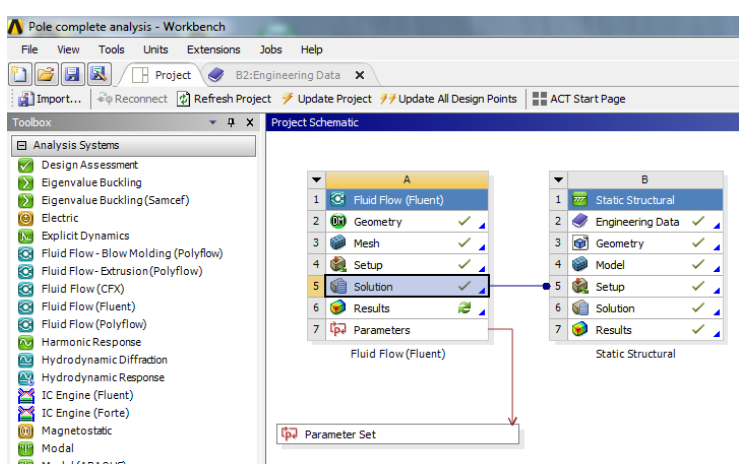

Fig. 4. FSI Window in Ansys Fluent

The ANSYS Structural Analysis is performed to have an accurate result obtained for the deflection caused by the wind velocity. The value obtained is 217 $\mathrm{mm}$, and the mathematical calculation yield a value of $224 \mathrm{~mm}$. The results obtained provide a good relation between the simulation and mathematical results, Figure 5.

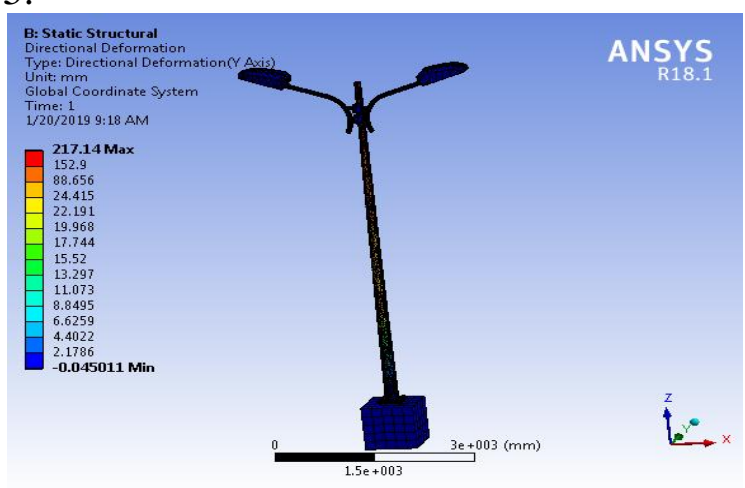

Fig. 5. The directional Deflection of the pole

The ANSYS have the capability to provide a more results in terms of Static structural Equivalent (Von Mises) stress. The stress obtained is $85 \mathrm{Mpa}$, Figure 6. 


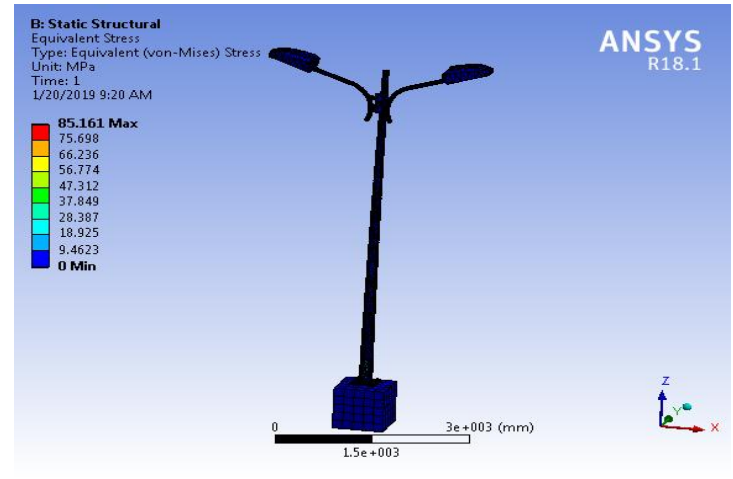

Fig.6. The equivalent (von-Mises) stress

\section{RESULT COMPARISON}

The results are obtained using the air velocity considered to be $200 \mathrm{Km} / \mathrm{hr}$, in Table 1.

TABLE 1: RESULTS COMPARISON

\begin{tabular}{|c|c|c|c|}
\hline \multicolumn{4}{|c|}{ Ansys versus Mathematical calculations } \\
\hline Results & $\begin{array}{c}\text { Force } \\
\text { exerted } \\
(\mathrm{N})\end{array}$ & $\begin{array}{c}\text { Deflection } \\
(\mathrm{mm})\end{array}$ & $\begin{array}{c}\text { Equivalent } \\
\text { Stress } \\
(\mathrm{MPa})\end{array}$ \\
\hline ANSYS & 1173 & 217 & 85 \\
\hline $\begin{array}{c}\text { Mathematical } \\
\text { Calculation }\end{array}$ & 1210 & 224 & Nil \\
\hline
\end{tabular}

\section{CONCLUSION}

The purpose of this research paper is to have a comparison between the mathematical and CFD simulation based study. The values of both methods are have a similar values, which indicates the simulation driven analysis can be adopted to such a kind of problems in future studies. The material used is also had a great impact over the simulation and its results. This research was done by considering the pole and its deflection only. The future work can be implemented by including the others parts of the street light such as (extender, illuminator). This work can also be further taken by implementing the solar panel, and thereby making the pole a self-sufficient. The simulation can be further taken ahead, by including the different direction of air inlet. This will provide a complete study of pole in different wind direction.

\section{REFERENCES}

[1] Sulthan Erfemli Gunaslan, Abdulhalim Karasin and M.Emin Oncu (2014). Properties of FRP material for Strengthening, page 656-660

[2] Cagri Uzay, Mete Han Boztepe and Needet geren (2016). Impact Energy Absorption capapcity of fiber reinforced polymer matrix ( FRP ) composites. Page no. 211-219

[3] User, S. (n.d.). Home. Retrieved April 28, 2019, from https://www.autonational.com/filamentwinder/markets/utility-poles.html

[4] Campobasso, M., Yan, M., Bonfiglioli, A., Gigante, F., Ferrari, L., Balduzzi, F., \& Bianchini, A. (2018). Low-speed preconditioning for strongly coupled integration of Reynolds-averaged Navier-Stokes equations and two-equation turbulence models. Aerospace Science and Technology 77, 286-298. doi:10.1016/j.ast.2018.03.015

[5] http://alshair.com.sa/index.php/subsidiaries/ind ustrial-division/shairco-fiberglass.Website Title AlShair Group, Article Title Al Shair Group, Date Accessed January 10, 2019

[6] http://www.performancecomposites.com/aboutcomposites-technical-info/122-designing-withfiberglass.html Website Title Fiberglass and Composite Material Design Guide Article Title performance composites Date Accessed January 10, 2019

[7] Versteeg, H. K., \& Malalasekera, W. (2011). An introduction to computational fluid dynamics: The finite volume method. Harlow: Pearson Education

[8] Yen, Tey Wah, et al. "Progress in Energy and Environment." Governing Equations in Computational Fluid Dynamics: Derivations and A Recent Review, vol. 1, 27 May 2017, pp. $1-19$.

[9] Bai, C.J. and Wang, W.C. "Review of computational and experimental approaches to analysis of aerodynamic performance in horizontal-axis wind turbines (HAWTs)." Renewable and Sustainable Energy Review 63 (2016) 506-519.

[10] Yang, J., Preidikman, S. and Balaras, E. "A strong coupling scheme for fluid-structure interaction problems in viscous incompressible flows." In: International Conference on Computational Methods for Coupled Problems in Science and Engineering, Barcelona, 2005.

[11] Cengel, Y. and Cimbala, J. Fluid Mechanics: Fundamentals and Applications. McGraw Hill Education, 2014. 
[12] Ferziger, J. H., \& Peric, M. (2002). Computational methods for fluid dynamics. Berlin Heidelberg: Springer Berlin Heidelberg

[13] Thabet, S., \& Thabit, T. H. (2018). CFD Simulation of the Air Flow around a Car Model (Ahmed Body). International Journal of Scientific and Research Publications (IJSRP), 8(7). doi:10.29322/ijsrp.8.7.2018.p7979

[14] P.Kannaiah, Design Of Machine Elements (Scitech Publications Pvt Ltd, 2006)
[15] Ibrahim, S. (2013). Design analysis and Commissioning Of High Mast Lighting Poles. IOSR Journal of Mechanical and Civil Engineering, 8(6), 40-46. doi:10.9790/16840864046

[16] Bansal, R. K. (2015). A textbook of strength of materials: Mechanics of solids ; in S.I. units. New Delhi: Laxmi Publications

[17] Cantilever Beams - Moments and Deflections. (n.d.). Retrieved from https://www.engineeringtoolbox.com/cantilever -beams-d_1848.html

[18] Goodno, B. J., \& Gere, J. M. (2018). Mechanics of materials. Boston, MA: Cengage Learning. 